\title{
Profiling Endosperm Purity of Commercial Mill Streams Preceded by Debranning Using Quantitative Chemical Imaging ${ }^{1,2}$
}

\section{D. Boatwright \\ Department of Biochemistry \& Molecular Biophysics and Microbeam Molecular Spectroscopy Laboratory, Kansas State University, Manhattan, KS, U.S.A.}

\author{
E. S. Posner \\ ESP International, Savyon, Israel
}

\section{R. Lopes}

Bunge Ltd., Brazil

\section{L. Wetzel ${ }^{3}$ \\ Microbeam Molecular Spectroscopy Laboratory, Kansas State University, Manhattan, KS, U.S.A.}

A brasive debranning is commonly used with hulled grains, such as rice, to remove the outer hull or husk and produce, for example, "polished" white rice (2). At present, preceding the break system with debranning equipment is not commonplace in wheat milling. The increased energy and capital costs of the hardware are issues that require economic justification based on overall mill efficiency and product purity considerations. However, a significant capacity increase in existing mill equipment, with appropriate changes in the flow sheet, can result in a reduction in energy costs ( $\mathrm{kWhr} /$ ton of wheat) with use of prebreak debranning. Hard wheat milling is one area in which adaptation of the milling system to debran the wheat grain prior to milling is an option that reportedly results in a practical economic pay back (9). The ideal debranning operation in hard wheat milling would evenly remove the outer layers of the pericarp without removing any endosperm or breaking kernels. Potential benefits include the removal of harmful elements from the kernel surface, flour streams with higher "brightness," and increased flour extraction and mill capacity (3).

Direct endosperm purity assessment of individual mill streams using a chemical imaging technique has been described previ-

\footnotetext{
${ }^{1}$ Contribution No. 16-011-J of the Kansas Agricultural Experiment Station, Manhattan, KS, U.S.A.

${ }^{2}$ In the graphic at the top of the page, successive individual flour stream warm color images (left to right) show declining endosperm purity from 99.5 to $88.0 \%$.

${ }^{3}$ Corresponding author. E-mail: wetzellab@ksu.edu, Tel: +1.785.532.6731; Fax: +1.785.532.4017.

http://dx.doi.org/10.1094/CFW-60-5-0211

(2015 AACC International, Inc
}

ously $(1,11,12)$. Previous studies were restricted to individual unit processes, such as an individual purifier, laboratory table-top milling study of the break system, or ranking flour stream purity for an experimental pilot mill. Laboratory sieving of postbreak fractions has also been reported $(4,5)$. In this article, we report on the endosperm purity profiles of 29 flour streams from a commercial flour mill running at a capacity of 204 metric tons $/ 24 \mathrm{hr}$ in which the break system is preceded by abrasive debranners (3). Our investigation team included the head operative miller on-site, who shouldered the responsibility for each operational parameter setting and supervised the stopwatch-timed collection of each of the product streams for weighing and various quality analyses. The specimens were produced on-site in Brazil during a routine commercial production process and were sent by air courier to the Kansas State University Microbeam Molecular Spectroscopy Laboratory, where endosperm purity was determined using quantitative chemical imaging with a research model imaging spectrometer (7).

We believe that this is the first reported wheat flour endosperm purity profile for a common wheat commercial milling operation in which a debranning process precedes the break system. The goal was to use the best possible chemical definition of wheat endosperm purity to produce this profile. Traditionally, the miller is concerned with the "brightness" of flour and considers a low ash value as being a good attribute. However, flour brightness involves only the part of the electromagnetic spectrum that the human eye can see. In contrast, chemical endosperm purity profiling is selective for endosperm in the presence of nonendosperm, and thus, a binary chemical mixture is assessed for purity with respect to endosperm versus nonendosperm.

In the case reported here, endosperm purity is directly assessed for 29 individual product streams from a commercial wheat mill in which abrasive debranning is a pretreatment. Our purpose is to objectively measure, calculate, and present the numerical endosperm purity value multiplied by the yield of the most pure stream first and subsequently add the cumulative contribution of each successive, slightly less pure mill stream. As each successive stream is added, the overall yield increases, and a slight degradation in composite endosperm purity occurs. The result is an endosperm profile that enables the miller to determine the point at which a cutoff is required to maintain the purity specifications required by their customer. The chemical composition of the endosperm and nonendosperm is determined based on quantitative chemical imaging using individual pixels. Each pixel in the image results from an individual near infrared spectrum representing the chemical composition of that pixel $(7,8)$. 
This method provides a totally objective measurement in which 81,920 spectra from the field of view produce chemically defined individual analyses. These analyses are tabulated to produce an objective flour stream endosperm purity value based on chemical structure. The subjective visual appearance of flour "brightness" from any given stream or mixture is produced from a bulk reflection response rather than from that of individual image pixels. The goal of this study was to apply the objective chemically defined pixel-counting method for assessing endosperm purity, with the expectation that the brightness from bulk color measurement would represent an essentially parallel assessment of purity. Pure endosperm, as defined chemically, is primarily composed of starch and protein. The presence of nonendosperm components in a field of view is indicative of incomplete separation of the wheat kernel endosperm from the bran, whereas ash determination measures only the inorganic (mineral) residue remaining after ignition. Application of the more precise partial least squares (PLS) data treatment to chemically define endosperm purity is described.

\section{Experimental}

Commercial Wheat Mill. A commercial wheat mill in Brazil with a capacity of 204 metric tons/24 hr and operating on three shifts (Fig. 1) was the source of all flour streams analyzed in this experiment. The grist for the experiment was from two Brazilian wheat varieties, Quartzo (70\%) and Supera (30\%). Both varieties are grown in Brazil on a regular basis, and therefore, the raw material analyzed was typical of the flour produced for relatively local consumption. The two abrasive debranner units (VTA model 10AB-L, Satake Corporation) installed prior to the double-high first and second break rolls had been in operation for $\approx 18$ months and adjusted to maximize efficiency during that time. A vertically mounted set of stones produced the abrasive action required to remove the bran. The debranning step was positioned prior to the break system in an attempt to achieve a brighter product at an improved extraction rate. A brighter final flour generally is more appealing to the customer and obviously constitutes high purity with respect to the removal of bran. Addition of the debranning capability has increased mill produc-
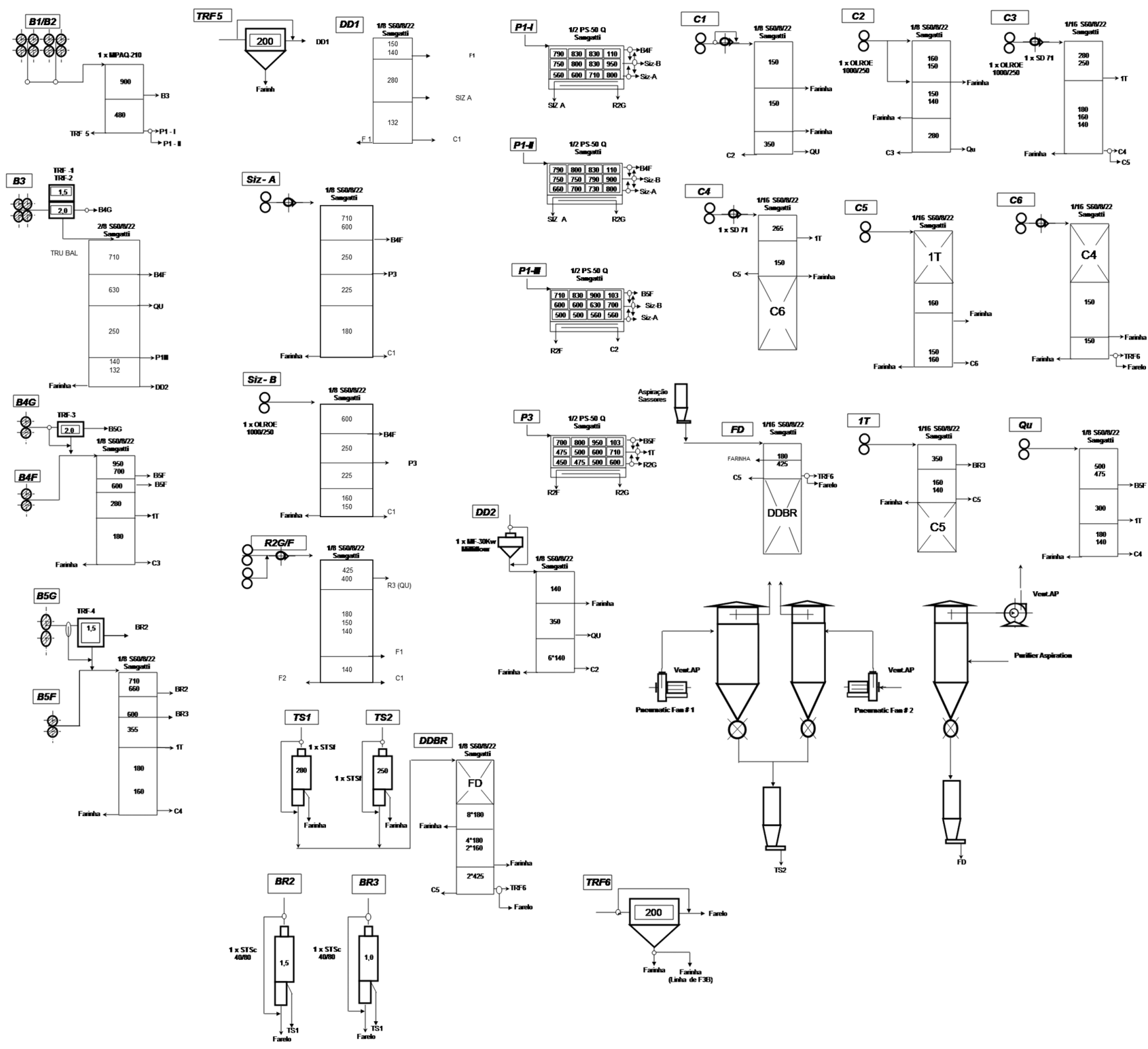

Fig. 1. Flow sheet for the 204 metric tons/24 hr capacity commercial wheat flour mill equipped with abrasive debranning technology. 
tion by $13 \%$. Under typical operating conditions, $4-6 \%$ of the total kernel weight is removed from the incoming wheat. Abrasion is applied directly to the cheeks of each wheat kernel, representing $\approx 80 \%$ of the total bran content of an individual kernel. Essentially, abrasive debranning results in removal of all of the outer and inner pericarp and, to a reasonable extent, most of the testa of the exposed portion of the wheat kernel (6).

Flour Specimens. Wheat flour specimens were collected from each product stream during operation of the mill on a regular production shift. The break release was set to $40 \%$ on first break and $70 \%$ on second break for the double-high break roll. The time of collection was monitored with a stopwatch, so the contents of each sample container would represent the quantity for a specific time interval and could be weighed to reflect the flow rate at that particular position, in addition to the corresponding purity determination. Twenty-nine mill streams were sampled to produce flour specimens for subsequent endosperm purity assessment in the analytical research lab at Kansas State University (Manhattan, KS). In addition to collection of flour from 29 individual product streams and replicates retained for local measurement of color and ash, spectroscopic standards were obtained. Bran specimens obtained from the debranning process and the very purest endosperm from the first midds were used as standards to calibrate the quantitative imaging method with respect to the raw materials and were contrasted with the waste material removed by the debranner and the purest endosperm product from this new mill operation.

Instrumentation. A near infrared imaging system (Sapphire model, Malvern Instruments Ltd.) that provides 81,920 near infrared spectra per field of view was used to acquire spectral data cubes for each flour sample. Data for each sample were collected in triplicate. The operation of this instrument with respect to individual intermediate and flour mill streams has been described previously $(1,12)$. The near infrared imaging spectrometer, which is equipped with four quartz tungsten halogen source lamps, employs a rectangular thermoelectrically cooled array of indium antimonide detector elements. A liquid crystal tunable filter provides electronic wavelength switching that enables simultaneous spectral acquisition at each $\mathrm{x}, \mathrm{y}$ coordinate (pixel) in the detector array and has no moving parts. The associated software controls optical data acquisition after establishing the maximum reflectance when focusing on the surface of a ceramic standard and obtaining the dark current value with no object at the focal point of the quartz objective. Before spectral acquisition, the granular sample material is placed in a metal planchette and covered with a 1 in. $\times 1.5$ in. glass microscope slide. Within the 1,200-2,400 $\mathrm{nm}$ wavelength range, select segments were scanned. A scanning step size of $3 \mathrm{~nm}$ was used to limit acquisition stare time, while providing adequate spectral resolution to discriminate pure endosperm from nonendosperm.

For brightness measurements, a reflectance colorimeter (CR410 Chroma Meter, Konica Minolta) equipped with a pulsed xenon flash lamp, optical fiber conductor, and diffusing elements was used to illuminate the circular target area $(50 \mathrm{~mm})$. Reflected radiation at 90 degrees from the specimen surface was transmitted to six silicon photodiode detectors. The repeatability standard deviation specification was 0.07 AU. The 1931 CIE defined color space (10) responses of three wavelength spectral features for colorimetry were closely matched. All flour streams have a similar brightness because in highly white specimens, where the optical response slope is small, discrimination between similar flour streams is a photometric challenge. Visible color ( $L^{\star}$ value) was measured on-site using the colorimeter to obtain a numerical index of brightness that matches consumer expectations. A more sophisticated reflectance spectrometer marketed by the same manufacturer, as well as other vendors, that could potentially be applied to color measurement provides a $10 \mathrm{~nm}$ bandpass, with a xenon flash lamp source, fixed spectrograph, and 60 element silicon photodiode array. Nevertheless, the visible absorption bands of wheat bran are broad.

Broadband electronic spectra phenomena and vibrational spectra were contrasted. The fundamental distinction between the spectral forms is that broadband color results from excitation of electrons. In contrast, the vibrational motion of chemically bonded atoms reveals molecular structural features. Thus, using first principles, the vibrational features allow chemical discrimination. It is fortuitous that the rate of change in the log $(1 /$ reflectance) that accompanies the change in chemical composition is readily measured by the image pixel. Chemical heterogeneity is revealed in the image and allows mathematical summation of values within the field of view. The slope of the cumulative endosperm purity curve and the endosperm contribution in each flour stream allows computation of the net purity achieved by selective exclusion of one or more inferior flour streams.

Quantitative Near Infrared Imaging Procedure. The difference in chemical structure between endosperm and nonendosperm content was used to sort and identify the pixels in each image spectroscopically. For each pixel, a near infrared spectrum was produced in $3 \mathrm{~nm}$ steps from 1,650 to $1,788 \mathrm{~nm}$ and from 2,150 to $2,228 \mathrm{~nm}$. If a simple binary designation of 1 for endosperm and 0 for $<0.5$ endosperm is used, the arithmetic amounts to simply counting the pixels of endosperm and dividing by the total number of pixels. The area targeted for analysis was $12.81 \mathrm{~mm} \times 10.24 \mathrm{~mm}$, resulting in a pixel size of $40 \mu \mathrm{m}$. The raw image intensity of each image pixel was first converted to absorbance, which describes optical density. The spectra were then baseline corrected and normalized.

We routinely use a PLS data treatment in which the binary designation is replaced by assigning an intermediate value to each of the 81,920 pixels in the field of view analyzed. In our previous experiments, careful establishment of the purity (endosperm) standard was selectively acquired for this purpose, and the impurity (nonendosperm) standard was spectroscopically defined by clean bran. PLS classification according to spectral libraries (more than 240,000 spectra for each component) defining endosperm and nonendosperm was applied to determine a pure endosperm multivariate identity reflected by the $\mathrm{z}$-axis value for each pixel. The intensity limits from 1 to 0 for endosperm and nonendosperm content correspond to warm and cool colors assigned to the maximum and minimum, respectively, of the scale. Experience is required to subjectively critically examine the data and select the appropriate threshold value below which data are excluded. Whereas care must be taken to establish a subjective maximum purity standard and, in contrast, impurity standard for the bran, standardized data handling makes the subsequent routine calculations objective. It is possible with PLS data treatment to assign a specific endosperm percentage to each pixel and obtain a summation.

\section{Results and Discussion}

The individual flour streams, with their respective flow and extraction rates, are ranked in descending order of endosperm purity in Table I. Multiplication of endosperm purity for a specific flour stream by its corresponding flow rate produces the 
Table I. Flour mill streams ranked based on endosperm purity

\begin{tabular}{|c|c|c|c|c|}
\hline Stream & Rank & $\%$ Endosperm & $\begin{array}{c}\text { Flow Rate } \\
(\mathbf{k g} / \mathbf{h r})\end{array}$ & $\begin{array}{c}\text { Extraction } \\
\text { Rate }\end{array}$ \\
\hline DD1 1 & 1 & 99.4 & 309.0 & 3.9 \\
\hline $\mathrm{DD} 1 \# 22 / 3$ & 2 & 96.3 & 261.0 & 3.3 \\
\hline DD1 2 & 3 & 95.8 & 82.5 & 1.0 \\
\hline SIZA 1 & 4 & 95.3 & 452.4 & 5.7 \\
\hline SIZB 1 & 5 & 94.9 & 150.0 & 1.9 \\
\hline DD1\#2 1 & 6 & 94.8 & 232.0 & 2.9 \\
\hline B3 & 7 & 94.4 & 315.0 & 4.0 \\
\hline $\mathrm{R} 21$ & 8 & 94.4 & 351.0 & 4.4 \\
\hline SIZA 2 & 9 & 94.1 & 2.4 & 0.0 \\
\hline C1 1 & 10 & 94.0 & $1,112.4$ & 14.0 \\
\hline $\mathrm{C} 22 / 3$ & 11 & 93.8 & 21.0 & 0.3 \\
\hline SIZAF 1 & 12 & 93.2 & 366.0 & 4.6 \\
\hline C1 2 & 13 & 93.1 & 17.6 & 0.2 \\
\hline TS2 & 14 & 92.6 & 54.0 & 0.7 \\
\hline SIZB 2 & 15 & 92.4 & 41.0 & 0.5 \\
\hline C1 3 & 16 & 92.3 & 8.1 & 0.1 \\
\hline B4 & 17 & 91.8 & 416.4 & 5.2 \\
\hline $\mathrm{C} 21$ & 18 & 91.8 & 370.2 & 4.6 \\
\hline DD2-1 & 19 & 91.6 & 337.5 & 4.2 \\
\hline C3 1 & 20 & 91.0 & 249.0 & 3.1 \\
\hline TRF06 & 21 & 91.0 & 27.0 & 0.3 \\
\hline TS1 & 22 & 90.5 & 117.0 & 1.5 \\
\hline C5 & 23 & 89.5 & 135.0 & 1.7 \\
\hline $\mathrm{C} 4$ & 24 & 89.2 & 81.0 & 1.0 \\
\hline $1 \mathrm{~T}$ & 25 & 88.7 & 132.0 & 1.7 \\
\hline QU & 26 & 87.7 & 113.0 & 1.4 \\
\hline C $32 / 3$ & 27 & 85.9 & 160.5 & 2.0 \\
\hline C6 & 28 & 81.9 & 82.5 & 1.0 \\
\hline DD2 $2 / 3$ & 29 & 81.2 & 16.1 & 0.2 \\
\hline
\end{tabular}

contribution of pure endosperm by the individual flour stream. The weighted combination of successive streams produces an endosperm purity profile in terms of descending purity and increasing yield. Cumulative endosperm purity is shown in Figure 2 based on select near infrared wavelength absorption data, which is dependent on the differences in chemical structure between the endosperm (analyte) and nonendosperm present in the flour matrix. The rate of change in the optical response is a function of the increased cumulative flour yield as inferior flour streams are added to the composite product. In contrast, the range of the broadband color measurements is severely limited (Fig. 3); however, in general, the response is parallel to the negative slope of the objective chemical values shown in Figure 2. The permutation of the broadband numerical values differs somewhat from the objective purity ranking based on contrasts in chemical structures. This apparent aberration was anticipated because of the broad bandwidth of $50 \mathrm{~nm}$ at the half height of the absorption band produced by the visible color filter with an appropriate wavelength maximum. Near infrared interference filter instruments typically have a $10 \mathrm{~nm}$ bandwidth at half height for each filter. For the liquid crystal tunable filter imaging spectrometer used to produce the direct endosperm purity profile, $3 \mathrm{~nm}$ wavelength increments are reported throughout the selected range.

Based on the premise that chemical structure is both an objective measure of endosperm purity and has a practical range, we elected to designate the quantitative near infrared imagebased data as the model. Select near infrared chemical images that highlight endosperm purity in warm (red) colors for individual flour streams are provided in Figure 4. These images extend over both the endosperm purity range (99.4-81.2\%) and the cumulative percent total product range illustrated in Figure 2. In contrast, the broadband color results that have a narrow

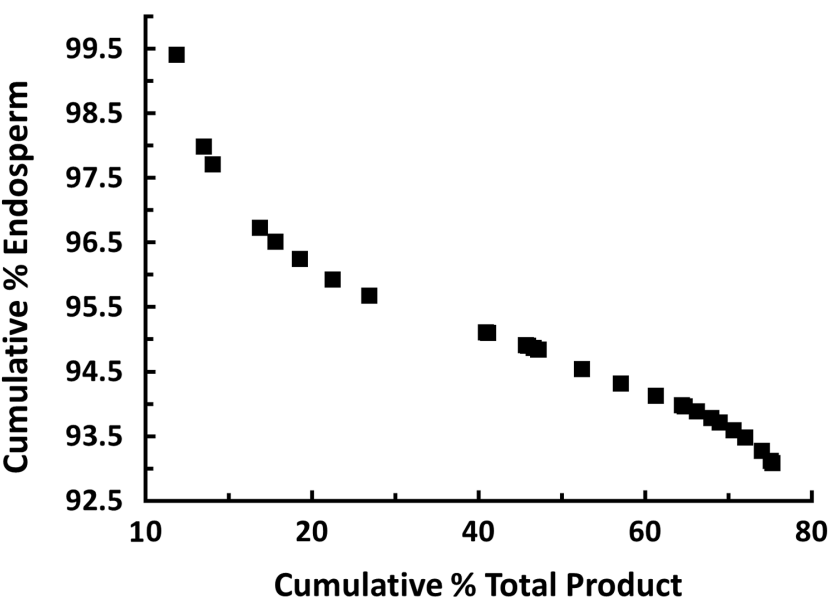

Fig. 2. Plot of cumulative percent endosperm versus cumulative percent total product. Decreasing endosperm purity is observed as more product streams are added.

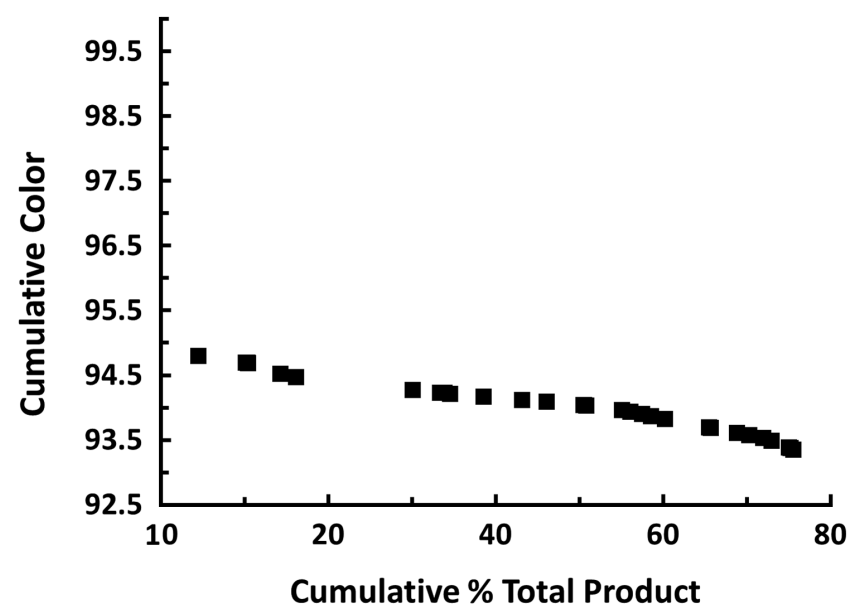

Fig. 3. A $92.5-100 \%$ reflection plot of cumulative broadband color scores versus cumulative percent total product. Compared with Figure 2, a smaller scale but similar slope of decreasing endosperm purity is observed as more product streams are added.

range, with sometimes overlapping values, produce a permutation inconsistent with the results of quantitative imaging. The permutation of color values (with their respective endosperm purity ranking), in order, was

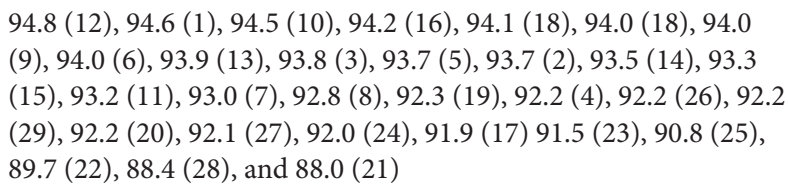

In the same sequence, the permutation of ash values (with their respective purity ranking), in order, was

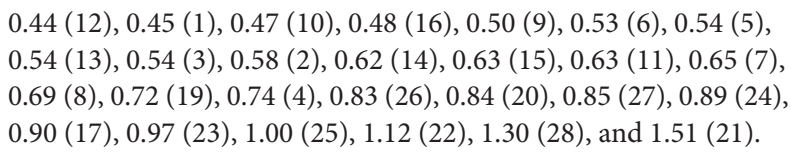

Although the ash content (mineral residue remaining after ignition) does not reflect the organic species present in the nonendosperm, aleurone, pericarp, germ, etc., the result of plotting ash versus endosperm purity produces a shotgun pattern with, as anticipated, a somewhat negative slope (Fig. 5). In the past, in areas where wheat was locally grown, milled, sold, and consumed, low ash content was a measure of the miller's resources and skill. How- 

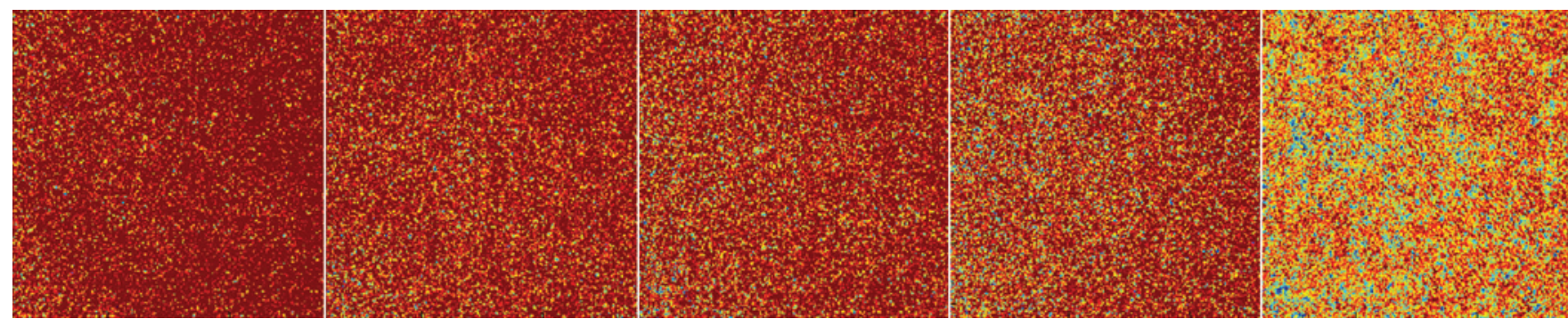

Fig. 4. Select near infrared chemical images highlighting the endosperm purity of individual flour mill streams. Left to right: DD1 1 (99.4\%), DD1\#2 1 (94.8\%), C1 1 (94.0\%), DD2-1 (91.6\%), and DD2 2/3 (81.2\%). Warm (red) colors are indicative of endosperm purity. Each image is representative of different points across the curve illustrated in Figure 2, including positions 1, 6, 10, 19, and 29, respectively.

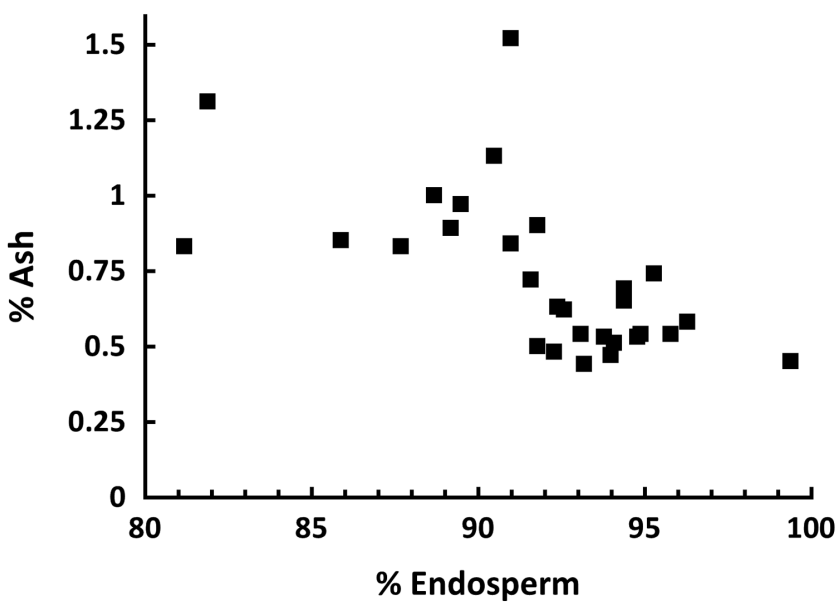

Fig. 5. Plot of percent ash versus percent endosperm resembling a random shotgun pattern.

ever, a large variation in the mineral content of the soil in which wheat is grown compromises ash as a meaningful indicator of flour impurity. Today, quantitative chemical imaging can be used to define endosperm purity. This new analytical capability provides a practical means to more accurately assess the result of a change in milling equipment or operating parameters. Broadband color or brightness incrementally decreases in concert with chemically defined endosperm purity, as indicated in Figures 2 and 3. The essentially parallel response of visible colorimetry enables an 8-12 hr shift miller to use visible colorimetry to indicate a short-term change in endosperm purity. However, chemically defined endosperm purity serves as a primary objective baseline.

From these data, a miller can make appropriate decisions to fill orders and meet specifications. The data also allow the miller to determine the penalty associated with adding mill streams in terms of decreasing endosperm purity. The cutoff point for streams with less endosperm reduces the overall yield; however, the endosperm purity index for traditional operation potentially allows future calculations for blending in which the purity number is retained but the flow rate is updated for a different run. The cumulative endosperm purities obtained for the milling system with debranning preceding milling and a traditional milling process are similar (11).

In the market for the products of the mill incorporating debranning, endosperm purity results in a $10-15 \%$ premium for materials with certain purity levels. This is similar to North American hard wheat mills that produce a ratio of patent to clear flour for different price considerations (9). In the case of debranning, the ability to designate and maintain high-value flour products that can be sold at a premium price affects the economic benefits derived through careful operation of a mill to obtain the best possible separation of endosperm from nonendosperm components.

\section{Conclusions}

Detailed quantitative chemical imaging offers a new method for determining what your wheat mill is producing in objective terms based on chemically defined endosperm purity. Unlike flour color, quantitative chemical imaging is not dependent on bran color. Unlike ash, the inorganic component of the outer layer of the kernel, it does not depend on the soil from which the wheat was grown.

\section{Acknowledgments}

The foresight and action of Virgil Smail (former KSU Grain Science Department head) in upgrading our laboratory's spectroscopic imaging capability is acknowledged. Chemical analysis of individual image pixels has resulted. We thank Bunge Ltd., Brazil for generous provision of mill stream specimens and Kansas Agricultural Experiment Station Project KS474 for financial support.

\section{References}

1. Boatwright, M. D., Gwirtz, J. A., Posner, E. S., and Wetzel, D. L. A quantitative near infrared imaging study of 1, 2, 3 break system endosperm yield from variation of $1 \mathrm{BK} / 2 \mathrm{BK}$ roll gap combinations. Int. Miller 3:35, 2013.

2. Dexter, J. E., and Wood, P. V. Recent application of debranning of wheat before milling. Trends Food Sci. Technol. 71(2):35, 1996.

3. Gregory, D. Debranning: A miller's perspective. Int. Miller Q. 1:37, 2010.

4. Gwirtz, J. A. New imaging method in milling: Near infrared focal plane array quantitative imaging of break stock part I. Milling J. 19(4):56, 2011.

5. Gwirtz, J. A. New imaging method in milling: Near infrared focal plane array quantitative imaging of break stock part II. Milling J. 20(1):52, 2012

6. Gys, W., Gebruers, K., Sorenson, J. F., Courtin, C. M., and Delcour, J. A. Debranning of wheat prior to milling reduces xylanase but not xylanase inhibitor activities in wholemeal and flour. J. Cereal Sci. 39:363, 2004

7. Lewis, E. N., and Haber, K. S. Hybrid-imaging spectrometer. U.S. Patent 09/828,281, 2002.

8. Lewis, E. N., Schoppelrei, J., and Lee, E. Near-infrared chemical imaging and the PAT initiative. Spectroscopy 19(4):26, 2004.

9. Posner, E. S., and Hibbs, A. N. Wheat Flour Milling, 2nd ed. AACC International, St. Paul, MN, 2005.

10. Smith, T., and Guild, J. The C.I.E. colorimetric standards and their use. Trans. Opt. Soc. 33:73, 1931.

11. Wetzel, D. L. Positive assessment of mill stream endosperm purity using chemical imaging. Cereal Foods World 58:133, 2013.

12. Wetzel, D. L., Posner, E. S., and Dogan, H. InSb focal plane array chemical imaging enables assessment of unit process efficiency for milling operation. Appl. Spectrosc. 64:1320, 2010. 


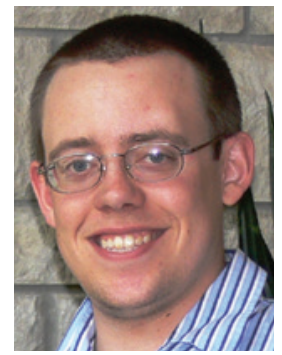

Mark D. Boatwright is a doctoral candidate in the Department of Biochemistry and Molecular Biophysics at Kansas State University. He was a chemistry option graduate in the Milling Science program. His preparation for a research career in agriculture was initiated from his encounters with the late Nobel Laureate Norman Borlaug at the World Food Prize Youth Institute. As a high school student, Mark was awarded a Borlaug-Ruan internship and spent two months performing research at the China Agricultural University in Beijing. His graduate research has involved infrared microspectroscopy with a focus on chemical imaging. Mark has presented talks at several national and international meetings and is the author of several publications. Mark is an AACCI member and can be reached at pitfall@ksu.edu.

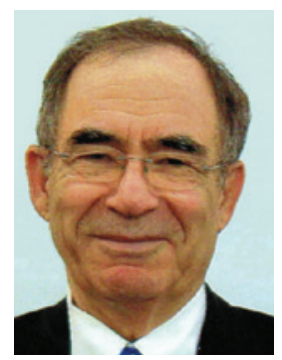

Elieser (Elie) S. Posner has been involved in the field of wheat flour milling for the past 55 years. He is a graduate of the Swiss Milling School and holds three degrees from the Grain Science Department at Kansas State University. Elie's practical experience includes milling machine construction, mill assembly, mill operation, mill management, mill analysis and performance improvement, academic research, and education. Elie has taught at KSU as an associate professor. In addition, he has authored and co-authored 39 refereed articles, 2 editions of the Wheat Flour Milling book published by AACCI, and 5 invited book chapters. He was also granted two U.S. patents. Elie is an AACCI member and can be reached at elie_posner@yahoo.com.

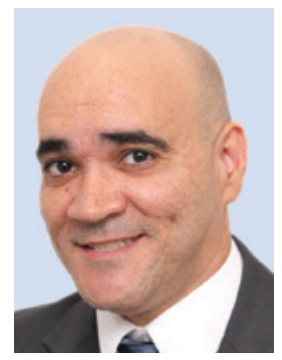

Ricardo Barroso Lopes is a master's student in the Food Engineering Department at the University of Ponta Grossa - Brazil. He received his bachelor's degree in chemistry at the same university. Ricardo has a deep in-terest in wheat mill building design, operational management, and process optimization. Throughout his professional life he has been involved in greenfield projects and the development of mill performance. Ricardo has presented talks at national meetings in Brazil and is the author of several publications. Ricardo can be reached at ricolopes2001@yahoo.com.br.

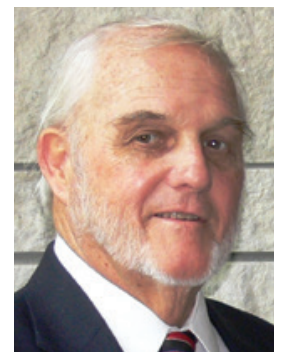

David L. Wetzel, a Ph.D. analytical chemist, returned to graduate school on an NSF Fellowship after several years of teaching college chemistry. He was recruited to the Grain Science Department from the University of Illinois (Springfield), where he taught instrumental analysis courses as an associate professor. As a KSU professor, his graduate students developed HPLC methods applied to cereal, while his technicians performed NIR. With the introduction of the first commercial research-grade infrared microscope, he became increasingly involved with FT-IR microspectroscopy and established the Microbeam Molecular Spectroscopy Laboratory in the KSU Chemistry Department. David has been published in 46 different journals and proceedings and has made more than 190 presentations at national and international conferences since entering the IR microspectroscopy field. David is an AACCI member and can be reached at wetzellab@ksu.edu.

\section{Erratum}

On pages 211 and 212 (text and Fig. 1 caption), references to the capacity of the commercial wheat flour mill have been corrected to 204 metric tons/24 hr. Changes to this article were made on November 19, 2015. 